\title{
Rendimiento de la citología por punción aspirativa transbronquial para el diagnóstico del compromiso mediastínico en el cáncer pulmonar
}

\author{
MAURICIO SALINAS F.*, MACARENA MUÑOZ A.**, ALFREDO JALILIE E.***, \\ MÓNICA ZAGOLIN B.****, SEBASTIÁN FERNÁNDEZ- BUSSY L.***, \\ CRISTINA FERNÁNDEZ F.*****, MAITE OYONARTE W.*****, DIONIS ISAMITT D.***, \\ CARLOS GUERRA P.***, KAREN CZISCHKE L.*** y SARA CHERNILO S.***
}

\author{
Utility of cytology by transbronchial needle aspiration for diagnosing \\ mediastinal nodal involvement in lung cancer staging
}

Introduction: Mediastinal nodal involvement is essential in lung cancer staging. This can be performed with transbronchial needle aspiration using a flexible bronchoscope. We present the experience of the Instituto Nacional del Tórax. Material and Methods: Retrospective study. The procedure was performed in patients with mediastinal nodes greater than $1 \mathrm{~cm}$ on computed tomography of the chest. Results: In 132 bronchoscopies, 136 transbronchial needle aspirations for cytological studies were performed, 98 (72\%) in subcarinal nodes and 38 (28\%) in other locations. In 64 (47.1\%) samples, lung cancer was diagnosed. In $100(73,5 \%)$ cases the final diagnosis was lung cancer with mediastinal involvement. In this series transbronchial needle aspiration had a sensitivity of $64 \%$ for lung cancer diagnosis. There were no complications. Conclusions: Transbronchial needle aspiration cytology is safe and useful for the diagnosis and staging of the mediastinum in lung cancer patients.

Key words: Biopsy, fine-needle, bronchoscopy, lung neoplasms, neoplasm staging.

\section{Resumen}

Introducción: Identificar el compromiso de los ganglios mediastínicos es fundamental en la etapificación del cáncer pulmonar. Esto puede hacerse con muestras citológicas obtenidas mediante punción aspirativa transbronquial. Presentamos la experiencia del Instituto Nacional del Tórax. Material y Método: Estudio retrospectivo. Se realizó el procedimiento en pacientes con ganglios mediastínicos mayores de $1 \mathrm{~cm}$ en la tomografia computada de tórax. Resultados: En 132 fibrobroncoscopías se realizaron 136 citologías por punción aspirativa transbronquial, 98 (72\%) en ganglios subcarinales y 38 (28\%) en otras estaciones. En 64 (47,1\%) muestras, se diagnosticó cáncer pulmonar. En 103 (76\%) casos el diagnóstico final fue cáncer pulmonar. En esta serie la citología por punción aspirativa transbronquial tuvo una sensibilidad de 64\% para diagnóstico de cáncer pulmonar. No se registraron complicaciones. Conclusiones: La citología por punción aspirativa transbronquial es una técnica segura y útil para la etapificación del mediastino en el cáncer pulmonar en nuestra experiencia.

Palabras clave: Biopsia con aguja fina, broncoscopía, neoplasias pulmonares, etapificación de neoplasias.

\footnotetext{
* $\quad$ Facultad de Medicina, Universidad de Chile.

** Facultad de Medicina, Universidad de Los Andes.

*** Unidad de Broncoscopías, Instituto Nacional del Tórax. Santiago de Chile.

**** Servicio de Anatomía Patológica, Instituto Nacional del Tórax. Santiago de Chile.
} 


\section{Introducción}

El cáncer pulmonar es la principal causa de muerte por cáncer en el mundo ${ }^{1,2}$. Se ha estimado que en 2008 murieron de cáncer pulmonar 1,4 millones de personas en el mundo entero ${ }^{2}$. En Chile, en 2010 fallecieron 2.774 personas a causa de esta condición ${ }^{3}$.

De acuerdo con la clasificación TNM del cáncer pulmonar, el compromiso de los ganglios mediastínicos es un elemento fundamental para la etapificación de los pacientes, permitiendo además establecer el pronóstico ${ }^{4}$.

Dado que la única opción curativa para los pacientes con enfermedad localizada es la cirugía resectiva, la selección de los casos debe hacerse en forma rigurosa ${ }^{1}$ y lo menos invasiva posible. La resecabilidad definida por la ausencia de compromiso mediastínico puede establecerse mediante mediastinoscopía, mediastinotomía o toracotomía ${ }^{1,5}$.

La punción aspirativa transbronquial o punción con aguja de Wang es un procedimiento diagnóstico alternativo, que puede realizarse ambulatoriamente, es menos invasiva y no se asocia a morbimortalidad significativa ${ }^{1,5}$; adicionalmente es un procedimiento de menor costo.

Schiepatti, un cirujano de tórax argentino, describió en 1949 el abordaje de los ganglios mediastínicos mediante la punción de la carina principal, utilizando un broncoscopio rígido ${ }^{6}$. En 1978 se utilizaron agujas flexibles a través de un fibrobroncoscopio para realizar punciones aspirativas transbronquiales y finalmente Wang propuso el procedimiento como una forma de etapificar el mediastino en el cáncer pulmonar ${ }^{6,7}$. En
2005 empezamos a realizar este procedimiento en la unidad de fibrobroncoscopías del Instituto Nacional del Tórax. A continuación describimos nuestra experiencia con este procedimiento.

\section{Materiales y Método}

En este estudio retrospectivo, se revisaron todos las fibrobroncoscopías en que se realizó punción aspirativa transbronquial entre enero de 2007 y abril de 2011. Se realizó el procedimiento en pacientes con cáncer pulmonar demostrado o altamente probable, que presentaban ganglios mediastínicos mayores de un centímetro en la tomografía axial computada de tórax. Las estaciones ganglionares a puncionar fueron escogidas en acuerdo con el radiólogo para determinar el mejor sitio de punción utilizando la clasificación de la Asociación Internacional para el Estudio del Cáncer Pulmonar (Figura 1).

\section{Procedimiento}

La fibrobroncoscopía fue realizada en la forma habitual, utilizando anestesia tópica con lidocaína y sedación consciente con midazolam y/o morfina. Siempre se tomó la precaución de realizar la punción aspirativa transbronquial antes de realizar otros procedimientos diagnósticos a fin de evitar la contaminación de las muestras ${ }^{6,7}$.

Se utilizó una aguja Conmed $21 \mathrm{G}$ en la mayoría de los casos. Para insertar la aguja, cada uno de los operadores utilizó alguna de las técnicas previamente descritas ${ }^{6,8}$ de acuerdo a su personal preferencia. En nuestra unidad se utiliza principalmente el método de "jabbing" que en español

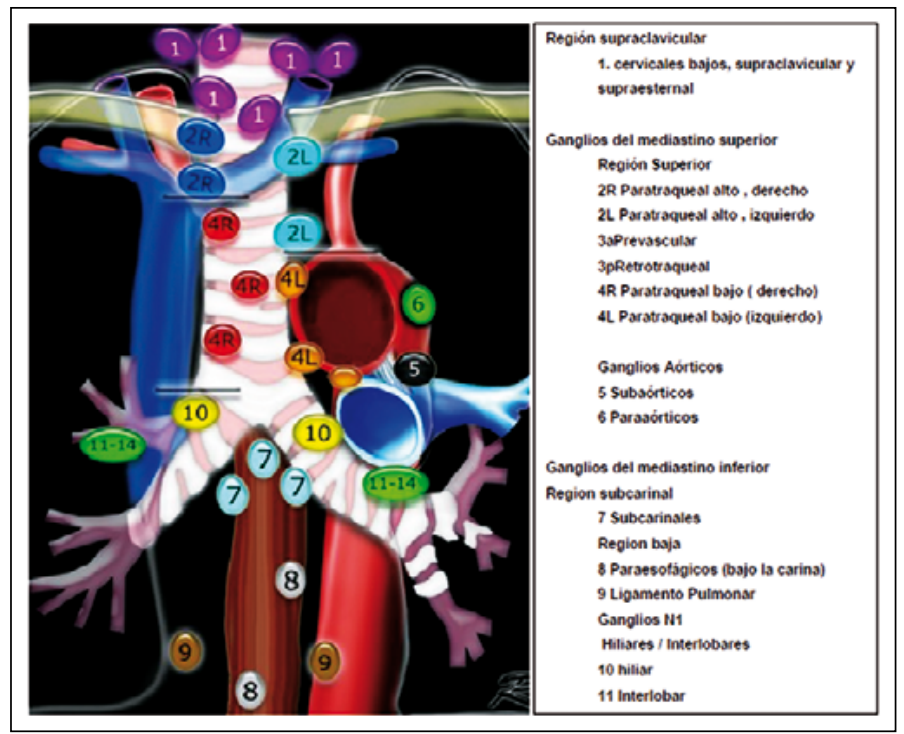

Figura 1. Mapa de los ganglios mediastínicos según la clasificación de 2009 de la International Association for the Study of Lung Cancer $^{8}$. Imagen de http://www.radiologyassistant.nl/ con permiso del autor. 
ha sido llamada "la técnica del pinchazo" o "punción del catéter-aguja a través del broncoscopio o bien el método de "hub-against-the-wall", que ha sido llamada en español la técnica de la lance$\operatorname{tada}^{6,8}$. Se realizó un mínimo de 2 y un máximo de 4 punciones, en cada estación ganglionar.

Excepcionalmente se contó con la asistencia del citopatólogo para la lectura inmediata de las muestras, que de resultar positivas hizo innecesaria la toma de muestras subsecuentes. Todos los pacientes firmaron un consentimiento informado antes del procedimiento.

\section{Análisis citopatológico}

Las muestras obtenidas fueron analizadas en forma diferida por patólogos institucionales que eran ciegos al resultado de otros exámenes cito o patológicos del caso. Las citologías fueron informadas como positivas para neoplasia, negativas para neoplasia, con otro diagnóstico (ejemplo: sarcoidosis) o de muestra inadecuada para el análisis.

\section{Análisis estadístico}

Los resultados se describen mediante promedios y desviación estándar para aquellas variables cuantitativas y utilizando frecuencias absolutas y relativas para aquellas categóricas.

Las citologías informadas positivas, se consideraron diagnósticas y verdaderos positivos para efectos del análisis.

En el caso de las citologías negativas para neoplasia el diagnóstico definitivo se obtuvo de distintas formas: mediastinoscopía, toracoscopía o seguimiento. En algunos casos no se logró confirmar o descartar el compromiso neoplásico del ganglio puncionado y en ese caso se asumió como falso negativo de la citología por punción aspirativa transbronquial, si el diagnóstico del paciente era cáncer.

Para otros diagnósticos (tuberculosis, neumonía, linfoma) el diagnóstico se obtuvo por, cultivos o inmunohistoquímica de otras muestras o por seguimiento del paciente.

En base a los criterios anteriores, se estimó sensibilidad, especificidad, valor predictivo positivo y negativo de la citología por punción aspirativa transbronquial, para el diagnóstico del compromiso mediastínico. El rendimiento se estimó sobre el total de estaciones ganglionares puncionadas. Se excluyeron de este análisis los sujetos sin diagnóstico definitivo confirmado.

Además se comparó el rendimiento diagnóstico entre las punciones a ganglios subcarinales y las otras estaciones, mediante prueba exacta de Fisher.
Las pruebas de significación estadística se realizaron a dos colas, con un alfa de 0,05.

El análisis estadístico se realizó en el programa Stata 11.0.

\section{Resultados}

Se realizaron un total de 132 fibrobroncoscopías, para 129 pacientes. La gran mayoría de ellas se realizó de forma ambulatoria. La edad promedio fue 63 años, setenta y tres $(56,5 \%)$ de ellos hombres. La mayor parte de las punciones fueron a la estación 7 (subcarinales) (Tabla 1).

En total se puncionaron 136 estaciones ganglionares. En 98 (72\%) de ellas el procedimiento fue en los ganglios subcarinales y las otras 38 (28\%) en las estaciones 4, 10 y 11, a derecha o a izquierda. En cuanto al resultado de las punciones, en $64(47,1 \%)$ muestras se obtuvo diagnóstico de cáncer pulmonar; en $58(42,6 \%)$ muestras no se logró diagnóstico y $14(10,3 \%)$ fueron insatisfactorias para análisis.

De las 72 muestras en donde no se obtuvo diagnóstico, en $39(54,2 \%)$ de ellas se diagnosticó cáncer de pulmón; de estas en 7 casos la cirugía confirmó compromiso ganglionar y en 3 casos la mediastinoscopía fue negativa, por lo cual fueron verdaderos negativos de la punción aspirativa transbronquial. En 12 (8,8\%) casos no se logró tener un diagnóstico definitivo.

El detalle del análisis del rendimiento diagnóstico se resume en la Tabla 2. En 103 (76\%) casos el diagnóstico final fue cáncer pulmonar,

Tabla 1. Características de la muestra

\begin{tabular}{|lrc|}
\hline & \multicolumn{2}{c|}{ Valor* } \\
Edad & $63 \pm 3$ años \\
Hombres & 73 & $(56,6 \%)$ \\
Estación ganglionar & $\mathbf{n}$ & $\mathbf{\%}$ \\
Paratraqueales (4R y 4L) & 10 & 7,7 \\
Subcarinales (7) & 98 & 72,1 \\
Hiliares (10) & 16 & 11,7 \\
Interlobares (11) & 4 & 2,9 \\
Otro (masas adyacentes) & 5 & 3,8 \\
No precisada & 3 & 2,2 \\
Diagnóstico del paciente & \multicolumn{2}{c}{} \\
Cáncer & 99 & 76,7 \\
Linfoma & 6 & 4,7 \\
Infecciones & 7 & 5,4 \\
Sarcoidosis & 2 & 1,6 \\
Otros & 3 & 2,3 \\
Sin diagnóstico & 12 & 9,3 \\
\hline
\end{tabular}

*Media ( \pm DE) para variables continuas; n (\%) para variables categóricas. DE: Desviación estándar. 
Tabla 2. Rendimiento diagnóstico de la citología por punción aspirativa transbronquial, según grupo ganglionar

\begin{tabular}{|lccc|}
\hline & Muestra total & Ganglios subcarinales & Otros grupos \\
Número de muestras & 103 & 75 & 28 \\
Verdaderos positivos (n) & 64 & 49 & 15 \\
Falsos positivos (n) & 0 & 0 & 0 \\
Verdaderos negativos (n) & 3 & 2 & 1 \\
Falsos negativos (n) & 36 & 24 & 12 \\
Sensibilidad (\%) & 64 & 67 & 56 \\
Especificidad (\%) & 100 & 100 & 100 \\
Valor predictivo positivo (\%) & 100 & 100 & 100 \\
Valor predictivo negativo (\%) & 7,7 & 7,7 & 7,7 \\
\hline
\end{tabular}

100 de ellos con compromiso del mediastino. En esta serie la punción aspirativa transbronquial tuvo una sensibilidad de $64 \%$ para identificar el compromiso mediastínico por cáncer pulmonar. La sensibilidad fue $67 \%$ en los ganglios sub carinales y $56 \%$ en otros grupos ganglionares, diferencia que no fue estadísticamente significativa.

No se registraron complicaciones graves o fatales en esta serie.

\section{Discusión}

En nuestro conocimiento esta sería la primera serie nacional publicada sobre citología por punción aspirativa transbronquial en la etapificación del cáncer pulmonar. Es un estudio retrospectivo con las limitaciones propias de este tipo de diseño; pero el número de pacientes estudiados es relevante y los resultados concuerdan con otras publicaciones, en cuanto a sensibilidad y rendimiento diagnóstico.

Harrow y cols ${ }^{1}$, demostraron una sensibilidad de $57 \%$ en un total de 360 pacientes estudiados. Dado que 273 de los 360 pacientes incluidos en ese estudio era inoperable, el $75 \%$ de los casos con citología negativa no fueron confirmados mediante cirugía. Los autores plantean que la sensibilidad de su estudio pudo fluctuar entre $57 \%$ en el peor de los escenarios y $91 \%$ en el mejor de los escenarios. Nuestro trabajo tiene las mismas limitaciones. En nuestro estudio no se pudo confirmar el compromiso ganglionar mediastínico en 29 casos con cáncer. Si todos ellos fueron verdaderos negativos la sensibilidad subiría hasta $90 \%$, que sería el mejor escenario; hay que hacer notar que la sensibilidad de $64 \%$ es el peor escenario, ya que se asume que todos esos casos no confirmados son falsos negativos de la citología.
En una revisión sistemática de la literatura ${ }^{5}$, sobre un total de 2.408 casos, la mediana de la sensibilidad del procedimiento fue $78 \%$, especificidad $100 \%$ y un VPP de $100 \%$. Existe gran variación en la sensibilidad publicada por los distintos autores, lo que es influenciado por distintos factores tales como: el tamaño y ubicación de los ganglios, el tipo de aguja utilizada, el número de aspiraciones realizadas, la disponibilidad de evaluación citológica inmediata y si se utilizan sistemas adicionales para guiar la punción como la ecografía ${ }^{5,6,7,9}$. Holty y cols. en un meta-análisis destacan que la sensibilidad depende críticamente de la prevalencia de compromiso tumoral del mediastino en la muestra ${ }^{10}$.

Un aspecto interesante es que distintos autores han destacado que esta técnica, está sub utiliza$\mathrm{da}^{7}$. Se estima que en Europa y en Estados Unidos entre el 11 y $30 \%$ de los neumólogos realiza el procedimiento9. Los argumentos esgrimidos para no realizar este procedimiento son dificultades técnicas, considerarla de escasa utilidad y la falta de un patólogo disponible para realizar la evaluación inmediata de las muestras ${ }^{9}$. Datos obtenidos por nuestro grupo en una encuesta aplicada a los neumólogos asistentes al $41^{\circ}$ Congreso Chileno de Enfermedades Respiratorias, concluyó que solamente el $9 \%$ de los broncoscopistas realizaba este procedimiento ${ }^{11}$.

Considerando que la punción transbronquial aspirativa es un procedimiento mucho menos invasivo que la mediastinoscopía, que puede realizarse en forma ambulatoria, tiene escasas complicaciones y es mucho más económica ${ }^{9,10}$, constituye una primera herramienta ideal para complementar a los estudios de imágenes y etapificar el mediastino en pacientes con cáncer pulmonar. Finalmente se ha destacado que la punción aspirativa transbronquial es un proce- 
dimiento altamente dependiente del operador, cuyo rendimiento mejora ostensiblemente con el entrenamiento $^{1,9}$. Confiamos en ello para mejorar nuestros resultados a futuro.

El estudio presentado tiene limitaciones. Un número importante de casos no se pudo confirmar o descartar el compromiso ganglionar por medios quirúrgicos, lo que es frecuente en el contexto de enfermedades neoplásicas, donde el estado del paciente o lo avanzado de su enfermedad no permite someter al sujeto a un procedimiento quirúrgico con un riesgo aceptable. Tampoco fue posible tener un registro confiable del tamaño de los ganglios y existe un número de sujetos que quedaron sin diagnóstico, fundamentalmente por falta de seguimiento. Pese a lo anterior, los rendimientos obtenidos están dentro de lo reportado en la literatura y las estimaciones de rendimiento diagnóstico asumen el peor escenario posible.

El estudio también tiene fortalezas. Todos los procedimientos fueron realizados en el mismo instituto, con un equipo de broncoscopistas entrenados y las biopsias leídas por patólogos experimentados, lo que da garantías respecto a la calidad e interpretación de los resultados.

En suma, el uso de citología por punción aspirativa transbronquial para el estudio del mediastino en el cáncer pulmonar es una herramienta de costo bajo, acceso fácil $\mathrm{y}$, rendimiento adecuado. Este estudio retrospectivo tiene limitaciones, pero el análisis de rendimiento diagnóstico es concordante con lo publicado, en una serie relevante de pacientes y demuestra que esta técnica permite ahorrar procedimientos quirúrgicos y costos.

\section{Bibliografía}

1.- HARROW E M, ABI-SALEH W, BLUM J, HARKIN T, GASPARINI S, ADDRIZZO-HARRIS D J, et al. The utility of transbronchial needle aspiration in the staging of bronchogenic carcinoma. Am J Respir Crit Care Med 2000; 161 (2 Pt 1): 601-7.

2.- JEMAL A, BRAY F, CENTER M M, FERLAY J, WARD E, FORMAN D. Global Cancer Statistics CA. Cancer J Clin 2011; 61: 69-90.

3.- Departamento de Estadística e Información de Salud del Ministerio de Salud de Chile. Series de defunciones por tumores malignos según edad 2000-2010. Disponible en http://www.deis.cl/estadisticas-mortalidad/

4.- DETTERBECK F C, POSTMUS P E, TANOUE L T. The stage classification of lung cancer: Diagnosis and management of lung cancer, $3^{\text {rd }}$ ed: American College of Chest Physicians evidence-based clinical practice guidelines. Chest 2013; 143 (5 Suppl): 191S-210S.

5.- SILVESTRI G, GONZÁLEZ A, JANTZ M, MARGOLIS M, GOULD M, TANOUE L, et al Methods for staging non-small cell lung cancer : Diagnosis and management of lung cancer, 3rd ed: American College of Chest Physicians evidence-based clinical practice guidelines. Chest 2013;143 (5 Suppl): 211S-50S.

6.- DISDIER VICENTE C, RODRÍGUEZ DE CASTROF. Punción transbronquial aspirativa. Arch Bronconeumol 2000; 36: 580-93.

7.- HERTH F J, EBERHARDT R. Flexible bronchoscopy and its role in the staging of non-small cell lung cancer. Clin Chest Med 2010; 31: 87-100.

8.- DASGUPTA A, MEHTA A C, WANG K P. Transbronchial needle aspiration. Semin Respir Crit Care Med 1997; 18: 571-81.

9.- GASPARINI S, SILVESTRI G A. Usefulness of transbronchial needle aspiration in evaluating patients with lung cancer. Thorax 2005; 60: 890-1.

10.- HOLTY J E, KUSCHNER W G, GOULD M K. Accuracy of transbronchial needle aspiration for mediastinal staging of non-small cell lung cancer: a meta-analysis. Thorax. 2005; 60: 949-55.

11.- OYONARTE M, CHERNILO S, CZISCHKE K, KAHN M, CAVADA G. Procedimientos de neumología intervencional: resultados de una encuesta realizada en el Congreso SER 2009. Rev Chil Enf Respir 2010; 26 : 217-8. (Resumen CL-19).

Correspondencia a:

Dr. Mauricio Salinas F

Email: mrsf24@hotmail.com

Dra. Sara Chernilo S.

Email: sarachernilo@yahoo.es

Instituto Nacional del Tórax

Avda. José M. Infante 717

Santiago-Chile. 